This is a postprint version of: Velasco, Juan Carlos and Wagner, Astrid. (2018). "Republikanische Identität für multikulturelle Gesellschaften. Von der Toleranz zur Anerkennung". Zeitschrift für philosophische Forschung, 68(3), 354-380.

\author{
Juan Carlos Velasco, Madrid \\ Astrid Wagner, Berlin
}

\title{
Republikanische Identität für multikulturelle Gesellschaften \\ Von der Toleranz zur Anerkennung
}

Die modernen Verfassungsstaaten wurden gebildet mit dem expliziten Ziel, die Koexistenz von Individuen und Gruppen mit unterschiedlichen Sitten, Überzeugungen und Lebensformen auf einem begrenzten Territorium rechtlich zu regeln. In historischer Perspektive stellen sie sich dar als juridisch-politische Mechanismen zur Sicherung des friedlichen Zusammenlebens in Gesellschaften, in denen die religiöse oder kulturelle Einheit durch Abspaltungen oder Zuwanderung zerbrochen war und disparate Überzeugungen unversöhnlich aufeinander trafen. Vereinbarungen zur verfahrensmäßigen Regelung der Konflikte waren notwendig. Von den Schriften Hobbes', Pufendorfs und Lockes bis heute hält die dominante Verfassungstheorie an den so etablierten Prinzipien fest. Doch der verfassungsmäßige Auftrag wurde in der Praxis keineswegs immer adäquat umgesetzt.

Durch Ansprüche kollektiver Identitäten auf Anerkennung kam es zu Spannungen im politischen Szenarium der Verfassungsstaaten. Die Forderungen seitens nationalistischer Bewegungen oder ethnischer Gruppen verschiedener Couleur haben sich in den letzten Jahrzehnten auf beiden Seiten des Atlantiks deutlich intensiviert. Die neuerliche öffentliche Relevanz ethnischer, nationaler, sprachlicher oder religiöser Differenzen zwischen Menschen, die auf einem Gebiet zusammenleben, ist eine Herausforderung für die liberale Politiktheorie und stellt die Rechtstheorie des Konstitutionalismus vor akute Probleme. Dies ist der Hintergrund für die prononcierte Frage des kanadischen Philosophen James Tully (I995, I): „Can a modern constitution recognise and accommodate cultural diversity?" ${ }^{\text {"I }} \mathrm{Um}$ sie beantworten zu können, ist

1 Das Buch von James Tully (1995), Strange multiplicity, Constitutionalism in an age of diversity, ist eine detaillierte Rekonstruktion des liberalen Konstitutionalismus. Der Autor zählt wie Charles Taylor, Will Kymlicka und Michael Ignatieff zu einer Gruppe zeitgenössischer kanadischer Philosophen, die inspiriert durch die separatistische Bewegung der Quebequois Theorie und Praxis des Liberalismus neu überdacht und eine Theorie kultureller Minderheiten ausgearbeitet haben. 
es hilfreich, die politischen Entwürfe zum Umgang mit dem beschriebenen Pluralismus nachzuzeichnen. In Abhängigkeit davon, ob die individuelle oder die kollektive Identität präferiert wird, lassen sich drei Gesellschaftsmodelle unterscheiden: erstens ein universalistisches Modell, der Liberalismus, demzufolge das politische Bündnis vor allem dazu dienen soll, die individuelle Identität zu gewährleisten, so dass der Schutz der persönlichen Autonomie zum zentralen Wert avanciert; zweitens ein eher partikularistisches oder kommunitaristisches Modell, das häufig nationalistische Züge trägt (das politische Bündnis dient in diesem Fall vor allem der Sicherung der üblicherweise ethnisch-kulturell definierten kommunalen Identität); und schließlich das republikanische Modell, das die politische Gemeinschaft als Ausdruck einer „bürgerlichen“ Identität versteht und von den Mitgliedern eine aktive Übereinkunft in öffentlichen Angelegenheiten fordert (die kollektive Selbstbestimmung stellt sich als Leitwert in diesem politischen Rahmen dar).

Dem Autor dieser Taxonomie, Ronald Beiner (2003, 32), zufolge sehen sich heutzutage viele Bürger „squeezed out between the opposing imperatives of liberalism and nationalism“. Der Ausweg aus dieser Disjunktion scheint nicht einfach. Besonders vom Nationalismus mit seiner symbolisch-kulturellen Dimension kann man sich nicht so einfach befreien. Religionen und Ideologien überlagernd, nimmt er den Status einer politischen Religion an. Auch den Liberalismus kann man nicht ablegen, ohne die politische Architektur unserer demokratischen Gesellschaften grundlegend umzugestalten. Das dritte Modell, das des Republikanismus, scheint auf den ersten Blick wenig hilfreich zur Überwindung des Dilemmas. Zumindest existieren kaum überzeugende Beispiele. Doch wenngleich Vertreter des Multikulturalismus wie Kymlicka (2003, 64f.) ausschließen, dass der Republikanismus eine plausible Alternative darstellen könnte, stellen die vorgebrachten Argumente kein Hindernis dar, die Gangbarkeit und möglichen Vorzüge dieses Weges zu prüfen. Der überraschende Wiederaufstieg des Republikanismus im letzten Jahrzehnt, zumindest in akademischen Kreisen, gibt Anlass zu einer solchen Prüfung. ${ }^{2}$ Und in der Tat finden sich unter den Neorepublikanern, die mit alten Ausprägungsformen der Tradition

2 Die Rehabilitierung des republikanischen Ideals ,ist, nach den Arbeiten von John Rawls, eine der wichtigsten theoretischen Errungenschaften im Bereich der politischen Philosophie“, deren Bedeutung vor allem auf ihrer Funktion als „Angelpunkt einer fruchtbaren Problematisierung der Prinzipien der liberalen Moderne“ (Savidan 2003, I5of.) beruht. 
gebrochen und gewisse liberale Lösungen übernommen haben, beachtenswerte Ansätze.

Das Ziel dieses Artikels besteht darin, die Möglichkeit eines identitätsstiftenden politischen Rahmens zu erwägen, der Freiraum lässt für kulturelle Unterschiede und sowohl normativ als auch institutionell über das Modell der passiven Toleranz des prozeduralen Liberalismus hinausgeht. Die Antithese von Liberalismus und Nationalismus zurücklassend (Konzeptionen, die von einigen Autoren keineswegs als unvereinbar angesehen werden, vgl. Tamir 1993 und Miller 1995) werden einige Aporien aufgezeigt, die bei der praktischen Umsetzung der beiden Modelle politischer Gemeinschaften zutage treten. Sie betreffen die Grenzen des Konstitutionalismus (I) und des Toleranz-Konzepts (2). Anschließend wird untersucht, ob und inwiefern ein republikanisches Modell für die komplexen modernen Gesellschaften einen Rahmen bereit stellen könnte, der für alle Mitglieder akzeptabel ist und Identifikationspotential bietet (3). Die Grundidee dabei ist, dass die Identifikation der Individuen mit dem Gesamtkomplex der Institutionen, des Rechtssystems und der politischen Praxis als Grundlage für die Ausbildung einer neuen Form von „Patriotismus" dienen könnte, sofern jene politische Partizipation und die Aufrechterhaltung kultureller Traditionen erlauben. Auf der Basis eines so verstandenen Patriotismus könnte sich eine offene und inklusive kollektive Identität ausbilden, wie sie für multinationale Gesellschaften wie Kanada, Belgien oder Spanien und mit Einschränkungen auch für durch Immigration entstandene multikulturelle Gesellschaften geeignet erscheint. Auf diese Weise, und nicht durch bloße liberale Toleranz, könnte den Ausdrucksformen der Differenz gebührend Raum gegeben werden.

\section{Der liberale Konstitutionalismus als verkappter Nationalismus}

Nach der Ära der großen Machtblöcke präsentiert sich der Liberalismus bzw. die liberale Demokratie als wichtigste ideologische Stütze der pluralistischen Gesellschaften. ${ }^{3}$ Abgesehen von historischen Gründen, ver-

3 Weit davon entfernt, eine homogene politische Tradition darzustellen, versammeln sich unter dem Etikett des Liberalismus sehr unterschiedliche, z. T. diskrepante Konzeptionen. Mit der Bezeichnung „demoliberal“ verweisen wir auf jene Tradition, die auf den demokratischen Ursprung des freiheitlichen Systems Nachdruck legt und sozusagen Rousseau mit Locke versöhnen will. Nicht der deregulierende 
dankt sich diese Vorrangstellung vor allem dem effektiven Zuschnitt der politischen Institutionen als isolierter und komplementärer Bereiche, so konzipiert, dass die Ausbildung von Machtmonopolen erschwert wird. Tatsächlich lässt sich der Liberalismus, um das schöne Bild von Michael Walzer $(1984,315)$ zu verwenden, verstehen als ,a certain way of drawing the map of the social and political world. [...] Liberalism is a world of walls, and each one creates a new liberty“. In diesem Sinne „stellt die Trennung von öffentlicher und privater Sphäre den Eckstein dar, auf dem die Organisation des Pluralismus der Kulturen, Identitäten und Lebensstile, der die modernen Gesellschaften auszeichnet, normativ aufruht" (Colom 1998, 196).

Die vom Liberalismus unterstützte Pluralität privater Interessen und Werte findet einen geeigneten Nährboden in der Neutralität des öffentlichen Raumes. ${ }^{4}$ Die Unparteilichkeit kann freilich niemals vollständig sein, da die öffentlichen Institutionen zumindest diejenigen verfahrensbezogenen Grundwerte übernehmen müssen, die den Pluralismus garantieren, z. B. den verfassungsmäßigen Schutz gewisser Freiheiten, Rechte und Chancen (vgl. Colom 1998, ig6 f.). Auf diese Weise nimmt der zunächst formale Liberalismus Züge einer, in Rawls' Worten, comprehensive doctrine an, die mit anderen ebenso umfassenden Entwürfen, z. B. den Religionen und politischen Ideologien, in Wettbewerb tritt. ${ }^{5}$ So wird der Pluralismus, dem der liberale Konstitutionalismus Raum zur Entfaltung gibt, im Grunde auf die für eine Gesellschaft charakteristische Heterogenität von Überzeugungen und Interessen beschränkt. Es ist bezeichnend, dass z. B. der von der spanischen Verfassung von 1978 als oberster

ökonomistische Diskurs des Laissez-faire soll hier als Referenz dienen, sondern jener andere liberale Diskurs, der die Autonomie des Individuums gegenüber der Gemeinschaft, die moralische Neutralität des Staates und das Prinzip der Gleichheit vor dem Gesetz betont. Sowohl Rawls als auch Dworkin, Raz und Habermas können als zeitgenössische Vertreter dieser politischen Tradition gelten.

4 Wenn man in liberalistischer Perspektive von Neutralität spricht, so ist damit gemeint, dass „basic institutions and public policy are not to be designed to favor any particular comprehensive doctrine" (Rawls I993, 194). Dworkin (1990, 4I) ist in diesem Punkt noch deutlicher: „Government must be neutral in ethics in the following sense. It must not forbid or reward any private activity on the ground that one set of substantive ethical values, one set of opinions about the best way to lead a life, is superior or inferior to others."

5 Seine eigene Fassung des Liberalismus stuft Rawls allerdings nicht als comprehensive doctrine ein, da sie sich auf den politischen Bereich als Referenz der Grundstruktur einer Gesellschaft und damit lediglich auf einen Teil des Gebietes der Moral beschränke (vgl. Rawls 2002, 38). 
Wert ausgezeichnete Pluralismus als „politischer Pluralismus“ (Art. I) beschrieben wird, mit explizitem Verweis auf die Repräsentationsfunktion der politischen Parteien, die „den politischen Pluralismus manifestieren“ (Art. 6). Dieser politische Plural ismus ist völlig kompatibel mit kultureller Uniformität und kann nicht mit einem religiösen, sprachlichen oder ethnokulturellen Pluralismus gleichgesetzt werden, der nicht nur die axiologische, sondern auch die epistemologische Ebene betrifft. Die staatliche Neutralität in politischen Angelegenheiten kann de facto Hand in Hand gehen mit der Förderung bestimmter kultureller Praktiken zu Ungunsten anderer, d. h. mit bestimmten Formen von Parteilichkeit.

Letzten Endes ist der kulturelle Pluralismus für den Liberalismus eine „unbequeme Art von Pluralismus“ (vgl. Requejo 2000, 44). So ist es nicht weiter erstaunlich, dass sich die Stellung des Konstitutionalismus (als wichtigste politisch-juridische Ausdrucksform des Liberalismus) zur kulturellen Vielfalt in den politischen Debatten als offene Frage darstellt. Für einige Autoren sind die Vorzüge des Konstitutionalismus im Umgang mit dem kulturellen Pluralismus offensichtlich. Paradigmatisch dafür ist die Auffassung von Ignatieff (200I, $32 \mathrm{f}$.): „A long-term solution requires an institutional setting in which the state is no longer communalized, no longer seen as the monopoly of any particular confessional, ethnic, or racial group, in which the state is reinvented as the arbiter of a civic pact among ethnic groups. Constitutionalism and the civic state are the institutional sine qua non of effective human rights protection in multiethnic states. Constitutionalism implies loosening up the unitary nation-state - one people, one nation, one state - so that it can respond adequately to the demands of minorities for protection of their linguistic and cultural heritage, and for their right to self-government." Dieser Gesichtspunkt lässt sich in dem lapidaren Satz zusammenfassen: „Democracy without constitutionalism is simply ethnic majority tyranny." (Ignatieff 200I, 30) Andere Autoren setzen kein so großes Vertrauen in den Konstitutionalismus: „Es ist nicht so, dass der Multikulturalismus keinen Platz findet im Konstitutionalismus, sondern dass [...] er nicht so recht zusammenpasst mit der spezifischen Form, in der sich letzterer entwickelt hat und die juridischen und politikphilosophischen Debatten beherrscht" (Cossío 2000, 75). Eine skeptische bis offen kritische Haltung zum klassischen Konstitutionalismus wird in den aktuellen politikphilosophischen Debatten vor allem von Charles Taylor und Will Kymlicka vertreten.

Der "demoliberale“ Konstitutionalismus geht davon aus, dass die politische Legitimität sich auf Abkommen zwischen den Mitgliedern einer 
Gesellschaft stützt. Betrachtet man die Bildung der modernen Nationalstaaten, erscheint dies eher als regulative Idee, welche bestenfalls diejenige Nation oder dominante ethnische Gruppe einbezieht, die als politisch aktives Subjekt verstanden wird. Minderheiten werden mehr oder weniger bewusst marginalisiert. In der Praxis geht man von einem homogenen Volk. Der Verfassungsstaat präsentiert sich als „Reich der Uniformität“: „The constitution founds an independent and self-governing nation state with a set of uniform legal and representative political institutions in which all citizens are treated equally, whether their association is considered to be a society of individuals, a nation or a community" (Tully 1995, 4I). Der Nationalstaat liberalen Zuschnitts soll, ausgehend von einer möglichst homogenen territorialen Machtverteilung, die Gesamtheit der Staatsbürger mit gleichen Rechten unter einer Oberhoheit vereinigen.

Dies spiegelt sich im strukturellen Zuschnitt der konstitutionellen Systeme wider. Die Verfassung ist als fundamentale und oberste Regel für die Ausübung öffentlicher Autorität erklärtermaßen einzige Quelle legitimer Macht (vgl. Tully 1995, 83). Der Verfassungstext wird explizit allen vorhergehenden Traditionen übergeordnet (vgl. Tully 1995, 85). Es wird außerdem, und dies ist entscheidend, eine homogene Gesellschaftsstruktur vorausgesetzt. Die genauere Bestimmung dieser Gesellschaft hängt von der dominanten politischen Ideologie ab: von den Liberalen wird sie als Assoziation privater und undifferenzierter Individuen definiert, von den Nationalisten als Gemeinschaft mit ethnisch-kulturellen Zügen und von den Kommunitaristen als Kollektiv, das sich durch eine einheitliche Auffassung des Gemeinwohls auszeichnet. An den einen wie den anderen Interpretationen lässt sich ,a marked tropism towards cultural [...] similarity" ablesen (Gellner 1987, 23). In allen Fällen handelt es sich um politische Projekte, die sich am Paradigma der Homogenität orientieren und folglich mit den faktisch gegebenen pluralistischen Verhältnissen kollidieren.

Die Forderungen, die mit einem kulturellen Pluralismus einhergehen, beschränken sich nicht auf die Anerkennung symbolischer Besonderheiten, sondern erstrecken sich auch auf die Anerkennung spezifischer Formen der Selbstverwaltung und Rechtsordnung. ${ }^{6}$ Der moderne Kons-

6 Neben den klassischen politischen Forderungen nationalistischer Bewegungen (nach eigenen Institutionen zur Gewährleistung der Autonomie, nach Selbstverwaltung und sogar nach Unabhängigkeit) finden sich ,interkulturelle “ Forderungen von Minderheiten. Sie betreffen in erster Linie den kritischen Punkt der Verwendung 
titutionalismus hat keine angemessenen Antworten darauf parat. In der Tat existierten in den Verfassungsordnungen bis vor sehr kurzer Zeit so gut wie keine Verfügungen, die ein Bestreben dokumentieren, die sozio-kulturelle Heterogenität anzuerkennen und ihr öffentliche Präsenz einzuräumen. Forderungen nach kultureller Anerkennung wurden ignoriert oder schlichtweg zurückgewiesen: von den Liberalen unter Berufung auf die kulturelle Neutralität des Staates, von den Nationalisten mit Verweis auf die Unverletzlichkeit der Integrität des Landes, von den Kommunitaristen im Rekurs auf einen sehr eng gefassten Begriff der sozialen Bindungen: „In each case, the demands are seen to be a threat to the unity of a constitutional association." (Tully 1995, 44) Angesichts der Forderung nach öffentlicher Intervention zur Erhaltung von Kulturen und Lebensformen erscheint der Egalitarismus nicht als angemessene Haltung. Ohne gezielte staatliche Vorkehrungen sind einige Minderheitskulturen langfristig dem Untergang geweiht. Die unterschiedlichen Umgangsformen mit dem Problem der Sprachenvielfalt und die verschiedenen Modelle territorialer Sonderregelungen in plurinationalen Staaten zeugen von der Komplexität der Aufgabe, den vielfältigen Ansprüchen gerecht zu werden.

Nichtsdestoweniger verstehen sich die meisten Verfassungsstaaten als Nationalstaaten mit unitarischem Charakter. Sie unterstützen die mehrheitliche Kultur durch machtvolle Instrumente der Sozialisation, insbesondere durch das öffentliche Bildungssystem. Weit entfernt von einer Neutralität in kulturellen Angelegenheiten, zählt die kulturelle Reproduktion der sozialen Mehrheit in vielen Fällen zu den expliziten Funktionen des Staates. ${ }^{7}$ De facto wird so die Kultur (die als national ausgezeichnete) zur Staatsangelegenheit. Selbstverständlich gibt es gro-

der Muttersprache in den Bereichen Erziehung, Ausbildung und sozialer Dienstleistungen, im öffentlichen Radio und Fernsehen sowie die kulturelle Prägung des Bildungsprogrammes in den Schulen. Darüber hinaus zählt die Einrichtung spezieller Foren zur Gewährleistung der politischen Repräsentation und Partizipation zu den typischen Forderungen kultureller Minderheiten (vgl. Tully 1995, I-6, u. Kymlicka 1996).

7 Üblicherweise behält sich der Staat entscheidende Kompetenzen im Hinblick auf die Gestaltung des Erziehungs- und Bildungssystems vor: Vereinheitlichung der Lehrprogramme, Genehmigung der Lehrbücher, Festlegung der Schulsprache. Ebenso kardinal ist seine Rolle bei der Festlegung der Feiertage, der staatlichen Symbole (Flagge, Hymne etc.) und der offiziellen Dokumente (Geburtsurkunde, Personalausweis, Reisepass, Schulzeugnisse, Militärpass etc.). Zu diesem Punkt vgl. Anderson 1993. 
ße Unterschiede zwischen dem kulturellen Engagement beispielsweise eines theokratischen Staates und dem eines Verfassungsstaates, der im Grundgesetz Religionsfreiheit garantiert und konfessionelle Unabhängigkeit proklamiert. Aber der staatliche Laizismus, d.h. die religiöse Neutralität des Staatsapparates, ist durchaus kompatibel z. B. mit der Förderung einer bestimmten Sprache, wodurch andere in ihrem Erhalt bedroht sind. ${ }^{8}$

Sobald eine bestimmte Kultur die Sphäre des Privaten und Unpolitischen überschreitet und nicht nur Toleranz, sondern öffentliche, staatliche Anerkennung oder gar allgemeine Verbindlichkeit eines speziellen Wertesystems einfordert, sind die Grenzen des vom liberalen Konstitutionalismus postulierten Neutralitätsprinzips in Frage gestellt. Dieses Problem bildet den Kern zahlreicher politischer Konflikte: erinnert sei hier an die endlosen Streitigkeiten über das Tragen des Tschador in öffentlichen Schulen in Frankreich, an die durch die Forderungen konservativer nordamerikanischer Bewegungen nach einem verpflichtenden Schulgebet verursachten Probleme, an den bis vor das Verfassungsgericht getragenen Kruzifix-Streit in Bayern, an die erbitterten Auseinandersetzungen um Abtreibung und um rechtliche Anerkennung der Homosexuellen-Ehe in den katholisch-dominierten Gesellschaften, oder auch an den offenen Protest, den so genannte Affirmative Actions, Maßnahmen zur Bevorzugung benachteiligter Gruppen, in multikulturellen Gesellschaften ausgelöst haben. Die Beispiele zeigen, dass das Politische nicht als ein Bereich konzipiert werden kann, in dem die symbolische Prägung der kollektiven Identitäten keine Rolle spielt. Damit aber sind die normativen Fundamente der klassischen Demokratietheorien liberaler Tradition grundlegend in Frage gestellt.

8 Zur identitätsstiftenden Funktion von Religion und Sprache vgl. Dion (1996, 19). Unabhängig von dem Wert, der einer jeden der beiden kulturellen Ausdrucksformen zugeschrieben wird, ist die Frage der Sprache von entscheidender Bedeutung, insofern sie die privilegierte Form der Übermittlung der Geschichte und Literatur einer Gesellschaft darstellt. Die historischen Erzählungen sind die wichtigsten Mittel zur Ausbildung und Erhaltung einer identitätsstiftenden Erinnerungskultur bzw. eines kollektiven Gedächtnisses. Aus diesem Grund existiert in den modernen Gesellschaften kaum ein Faktor von höherem Potential zur Stiftung einer kollektiven Identität als die Sprache. 


\section{Die strukturellen Grenzen der Toleranz}

„Toleranz sollte eigentlich nur eine vorübergehende Gesinnung sein: sie muß zur Anerkennung führen. Dulden heißt beleidigen."

(J. W. Goethe, Maximen und Reflexionen, \$875)

In liberalistischer Sicht ist die Verteidigung der individuellen Autonomie das wichtigste Ziel der politischen Gemeinschaft. Um es zu erreichen, konzentriert man sich vor allem auf zwei Prinzipien: die Garantie der individuellen Rechte und die Gleichheit vor dem Gesetz. Die Vertreter des Liberalismus gehen mehrheitlich davon aus, dass diese Prinzipien auch zur Lösung der mit der kulturellen Diversität einher gehenden Probleme einschlägig sind. Im Grunde müsse die Strategie, die der liberale Staat gegenüber religiösen Gruppen angewandt und die gute Ergebnisse für das Zusammenleben erzielt hat, nur auf die ethnokulturellen Konflikte übertragen werden. ${ }^{9}$ Die klassischen liberalen Prinzipien und Praktiken werden als Grundlage zur Etablierung gerechter Verhältnisse auch im Bereich der ethnokulturellen Beziehungen angesehen. Entsprechend besteht das Rezept der Liberalen im Umgang mit interkulturellen Konflikten in der Empfehlung, Toleranz zu üben nicht nur in den zwischenmenschlichen Beziehungen, sondern vor allem im öffentlichen Bereich. Toleranz als politische Tugend zu betrachten gehört mittlerweile zum Grundbestand der liberalistischen Doktrin.

Das historische Motiv, das zur Verbreitung der Toleranz-Praxis führte, ist wohlbekannt. Im frühneuzeitlichen Europa hatte keines der verschiedenen christlichen Bekenntnisse reale Aussichten auf die Durchsetzung einer sozialen Monopolstellung (vgl. Kamen 1987, Fetcher 1994, Forst 2003). So kam man zu der Überzeugung, dass Toleranz mehr zum sozialen Frieden beitragen könne als jeder Versuch, eine einzige staatliche Religion gegen den Widerstand der abtrünnigen Minderheiten durchzusetzen. Vergleichbare Argumentationen finden sich in den kanonischen Texten der Aufklärungszeit: John Lockes A Letter concerning Toleration (I689) und Voltaires Traité sur la tolérance (I763). ${ }^{10}$ Damals

9 Vgl. Kymlicka 200I, 50. Habermas (2005, 274) zufolge kann die Anerkennung des religiösen Pluralismus diese Vorbildfunktion übernehmen, weil sie auf exemplarische Weise den Anspruch von Minderheiten auf Inklusion zu Bewusstsein bringe.

10 Neben den bekannten Texten Lockes zur Verteidigung der Religionsfreiheit und der Schrift Voltaires gegen den religiösen Fanatismus entstand in der Aufklärungszeit ein vielleicht noch denkwürdigerer Text: Lessings Drama Nathan der Weise (I779). 
wie heute dient die Toleranz dazu, „eine pluralistische Gesellschaft davor [zu bewahren], als politisches Gemeinwesen durch weltanschauliche Konflikte zerrissen zu werden“ und „die gesellschaftliche Destruktivität eines unversöhnlich fortbestehenden Dissenses “ aufzufangen (Habermas 2005, 265 u. 269).

Die Konzeption von Toleranz, die sich zu Beginn der Neuzeit Durchbruch verschaffte, betonte den Wert der Diversität wohl wissend, dass die Spontaneität des sozialen Lebens nicht mehr ausreichte, um die bestehenden Differenzen zu überwinden. Es handelt sich um eine grundsätzlich pragmatische Perspektive: das kleinere Übel der Diversität wird akzeptiert, um das grössere Übel der Fortsetzung ziviler Konflikte zu vermeiden. Diese Argumentation findet sich bereits in Lockes Verteidigung der Toleranz. ${ }^{11}$ Allerdings ist die Idee der Toleranz nicht ursprünglich liberal. Sie hat ihre Wurzeln in der christlichen Tradition, und daher stammt eine besonders negative Konnotation, die dem Begriff noch heute anhaftet. In der christlichen Weltsicht steht die Toleranz für die Duldung einer Sünde, die man nicht verhindern kann oder will, unter Berufung auf das Recht der Wahrheit, die den Irrtum anerkennt, und der Tugend, die das Laster anerkennt. Sie stellt das Zulassen eines realen oder angenommenen Übels dar (permissio negativa mali). Die Bezeichnung der Bordelle als „Häuser der Toleranz“ illustriert beispielhaft diese Denkweise. Aber die Geschichte des Toleranzbegriffs ist nicht nur mit der Geschichte des Zusammenlebens unterschiedlicher Religionsgemeinschaften verbunden, sondern auch mit den aufklärerischen Bestrebungen zur Errichtung einer vom religiösen Bereich getrennten öffentlichen Sphäre. In den letzten beiden Jahrhunderten, vor allem im 20. Jhd., wurde darüber hinaus deutlich, dass die Keimzellen der Intoleranz nicht nur in den Religionen, sondern auch und vor allem in den Nationalis-

Dort wird der Begriff der Toleranz zwischen den monotheistischen Religionen anhand der "Parabel von den drei Ringen“ entwickelt, die schon seit dem Mittelalter zwischen spanischen Juden zirkulierte. Davon ausgehend, dass wir alle zunächst Menschen sind, und dann erst Jude, Moslem oder Christ, und dass niemand im exklusiven Besitz der Wahrheit ist, zeigt der Text, wie ein zivilisierter Streit zwischen den verschiedenen Positionen möglich ist, in dem jede Partei, ohne ihre eigenen Wertvorstellungen zu opfern, die anderen Parteien als Verfechter authentischer Wahrheiten anerkennt.

11 In unseren Tagen ist John Gray (200I) vielleicht derjenige Autor, der mit grösster Klarheit den liberalen Begriff der Toleranz als Modus vivendi, d. h. als Rahmen friedlichen Zusammenlebens zwischen unterschiedlichen Kulturen und Lebensweisen verteidigt hat. 
men zu verorten sind, deren anti-aufklärerisches und anti-humanistisches Wüten - paradigmatisch im Nationalsozialismus zum Ausdruck gebracht - die demokratischen Systeme zwingt, nach neuen Grundlagen des Zusammenlebens zu suchen. Dadurch hat sich das Aktionsfeld der Toleranz enorm erweitert.

Jenseits des historischen Kontexts ist es aufschlussreich, die semantische Struktur des Begriffs zu untersuchen. Eine Person handelt tolerant, wenn sie bereit ist, von einer Intervention abzusehen, die darauf gerichtet wäre, Verhaltensweisen anderer Personen, welche eigene relevante Überzeugungen verletzen, zu verbieten, obgleich sie dazu in der Lage wäre (vgl. Garzón Valdés 2000, I8I-198). Toleranz ist weder mit Indifferenz noch mit Resignation zu verwechseln, sondern setzt in einem ersten Schritt eine Haltung offener Ablehnung voraus. ${ }^{12}$ Die Toleranz-Akte sind unilateral, punktuell und widerrufbar. Sie hängen per definitionem vom freien Willen des Tolerierenden ab. Diese Zufälligkeit macht sie als Grundlage eines stabilen normativen Systems untauglich. In der Tat kann Toleranz als dispositionale Eigenschaft schwerlich von jedem Staatsbürger gefordert werden. Insofern erscheint sie nicht als geeigneter Weg, um das Zusammenleben in multikulturellen Gesellschaften zu regeln. Sie erscheint als gute Verfahrensweise in den zwischenmenschlichen Beziehungen, doch für den öffentlichen Bereich ungeeignet. Zur Arbeit eines Beamten in einem gerechten Staat gehört es weder zu dulden noch Kompromisse einzugehen noch zu tolerieren. Seine Aufgabe ist es, die den Individuen zustehenden Rechte anzuerkennen. Sein Verhalten sollte weder wohlwollend noch jovial, sondern unparteilich sein. Auch in Bezug auf Handlungen, die von einem Verbot ausgenommen wurden, sollte nicht von Toleranz die Rede sein; richtiger wäre es hier, von ausdrücklichen Autorisierungen oder Zulassungen zu sprechen, die Rechte zuerkennen, also eigentlich von Anerkennung.

Im Bereich der interpersonalen Beziehungen geht es vor allem darum, Unparteilichkeit zu gewährleisten und Willkür zu verhindern. Diese Funktion wird normalerweise durch das formale Recht erfüllt (Rechtssicherheit). Ein Beispiel für Toleranz im privaten Bereich wäre das eines Unternehmers, der seinem muslimischen Angestellten erlaubt, die Arbeitszeit zu unterbrechen, um vorschriftsmässig seine Gebetszeiten einzuhalten. Ein Beispiel für Intoleranz wäre das eines Barbesitzers,

12 Carlos Thiebaut $(1999,50)$ verwendet zur Beschreibung der Phasen des Toleranz-Aktes die geglückte Wendung bucle de negatividad, um eine Ablehnung zu bezeichnen, die sich reflexiv umkehrt. Vgl. auch Forst 2003, 30-52. 
der sich weigert, einen Kunden zu bedienen, weil dieser einer anderen Volks- oder Kulturgemeinschaft angehört. Ganz abgesehen davon, ob solche Handlungen lobenswert oder verwerflich sind, liegt es im Belieben desjenigen, der sich in überlegener Position befindet, zu entscheiden, ob er ein großzügiges Zugeständnis macht, sich eine Selbstbeschränkung auferlegt, oder nicht. Deshalb sollten diese Angelegenheiten nicht Gegenstand der Toleranz sein, sondern einer juristischen Regelung unterliegen. Wenngleich zur Lösung interkultureller Konflikte auf eine umsichtige Ausübung von Toleranz zu hoffen ist, so ist doch eine Haltung zu bevorzugen, die verhindert, dass Streitigkeiten sich vertiefen und das soziale Klima vergiften. So ist es angezeigt, den Terminus Toleranz nicht mehr in seiner ursprünglichen Bedeutung zu verwenden, sondern in dem heutzutage verbreiteten Sinn einer offenen, interessierten, aber nicht moralisch indifferenten und unkritischen Haltung im Dialog mit Andersdenkenden (vgl. Höffe 1999, 205 f.) und im Sinn der Fähigkeit zur Revision der eigenen Ideen, der „Kooperations- und Kompromissbereitschaft" (Habermas 2005, 268). John Rawls (1993, 217) geht noch einen Schritt weiter und konzipiert die Aufgabe einer politischen Einigung trotz grundlegender normativer Differenzen gar als staatsbürgerliche Pflicht, als ,duty of civility“.

Das Toleranz-Konzept des Liberalismus hat klar gesetzte Grenzen: unter dem Stichwort der Toleranz hat die liberale Kultur den Respekt der Identität der privaten Individuen nur insoweit gefördert, als damit kein Angriff auf die Freiheiten und Identitäten anderer verbunden ist. Der Respekt gegenüber der Pluralität entspricht dabei im Prinzip der öffentlichen Anerkennung derselben Würde aller privaten Identitäten. Gelegentlich aber fordert die Verpflichtung auf die Menschenrechte in der Praxis eine intolerante Haltung gegenüber jenen Lebensformen, die sich ihrerseits intolerant verhalten. Die von den Liberalen befürwortete staatliche Neutralität beinhaltet, auch wenn das paradox erscheinen mag, die Aufhebung des Prinzips der öffentlichen Nichteinmischung, wenn es um die Verteidigung der Freiheiten geht. Fundamentalismen und Fanatismen, die sich auf den kulturellen Pluralismus stützen, um ihn dann, sobald sie entsprechende soziale Machtpositionen erlangt haben, abzuschaffen, Positionen also, welche die Voraussetzungen des Konstitutionalismus untergraben, sind klar jenseits der Grenze des Tolerierbaren zu verorten. Keine Verletzung der Menschenrechte ist tolerierbar. In der Geschichte finden sich zahlreiche Beispiele kollektiver Lebensformen, deren Kultivierung oder Unterstützung nicht mit moralischen 
Gründen zu rechtfertigen ist. Eine tolerante Haltung ist nur gegenüber jenen Verhaltensformen angebracht, die die Grenzen des Akzeptablen und Diskutierbaren nicht überschreiten. Zur Bestimmung dieser Grenzen vgl. Wagner 2013.

Anstelle der gönnerhaften Haltung der Toleranz wäre es angebrachter, sich für die Anerkennung des Anderen und seines Rechts auf Alterität einzusetzen: „Gegenüber dem Anderssein ist zunächst die Vermeidung der Diskriminierung, also gleiche Achtung für jeden geboten - und nicht wie gegenüber dem Andersdenken Toleranz." (Habermas 2005, 265) Dies gilt nicht nur im Hinblick auf die religiösen Überzeugungen, sondern mutatis mutandis auch für die verschiedenen identitären oder kulturellen Besonderheiten, sofern sie dem friedlichen Zusammenleben nicht entgegenstehen. Die Anerkennung der Rechte macht die voluntaristische Toleranz in vielen Fällen überflüssig. „Die wahre Liberalität ist“, wie Goethe sagt, „Anerkennung“ (Maximen und Reflexionen, \$876). So ist Fetschers (1994) Auffassung von Toleranz als einer kleinen unentbehrlichen Tugend für die Demokratie nicht ohne weiteres zuzustimmen. Aus dem Lob der Toleranz als privater Tugend und ihrer historischen Rolle im Kampf um die Rechte des Individuums folgt nicht, dass sie auch als Grundlage für das gute Funktionieren demokratischer Institutionen anzusehen ist. ${ }^{13} \mathrm{Da}$ die multikulturelle Gesellschaft nicht aus sich heraus zur Harmonie zwischen den Parteien neigt, muss dies durch verlässlichere Mittel gesichert werden. Wenngleich Toleranz immer eine empfehlenswerte Haltung in einer demokratischen Gesellschaft sein wird, stellt die öffentliche Anerkennung der verschiedenen Kulturen, sowohl auf politischer wie auch auf juristischer Ebene einen weiteren wichtigen Schritt zur Etablierung politischer Gerechtigkeit und zur Garantie der individuellen Autonomie dar. ${ }^{14}$

13 Dass die Toleranz mit der Einrichtung des Verfassungsstaates ihren Anwendungsbereich schwinden sieht bedeutet nicht, dass ein Appell an diese Tugend sinnlos sei. Vor allem in der Ausübung von Rechten gilt es, dem Toleranzprinzip gemäss, die Konsequenzen zu beachten, die damit hinsichtlich der Rechte anderer verbunden sind. Vgl. das eindringliche Plädoyer Thiebauts (1999) für die Aktualität der Toleranz, nicht in ihrer negativen Form (als Ertragen des Andersartigen), sondern in ihrer positiven Fassung (als Verstehen des Anderen, „dem Anderen einen Platz in unserem Argumentationsraum einräumen").

14 Aus ähnlichen Gründe plädieren die Verfechter der „Politik der Differenz“ für eine Ersetzung der liberalen Tugend der Toleranz durch die öffentliche Anerkennung des Pluralismus (vgl. Taylor 1993). 


\section{Bürgerlicher Republikanismus und identitärer Pluralismus}

„Der Liberalismus fragmentiert. Der Kommunitarismus
isoliert. Der Republikanismus hingegen verbindet. Der
Erste konzipiert uns als souveräne Willen und Egoisten;
der Zweite als Stammeswesen. Nur der Dritte beruft sich
auf die wesentlich interaktive Natur jedes sozialen Le-
bens, und zwar ohne die Automie des Individuums oder
das Ausserhalb der Gemeinschaft Stehende abzulehnen."
(Giner 200o, I7I).

Kritikwürdig erscheint, dass die Institutionen und konstitutionellen Normen liberalen Zuschnitts häufig nur die kulturelle Identität der mehrheitlichen Gruppe widerspiegeln, ebenso wie die Berufung auf privatisierte Toleranz als elegante Form, die faktische Hegemonie der mehrheitlichen Kultur zu verstecken. So gilt es, alternative Lösungen vorzuschlagen, Auffassungen kollektiver Identität und Staatsbürgerschaftsmodelle zu entwickeln, die den politischen Rahmen für einen positiven Umgang mit dem Pluralismus der modernen Gesellschaften bilden können. Angesichts der resistenten monokulturellen Gestaltung vieler multikultureller Staaten und fehlender sozialer Einbindungen geht es darum, Muster einer kollektiven Identität zu finden, die integrative und inklusive Züge trägt.

Jede Art kollektiver Identität, die monokulturell definiert ist, negiert die Transkulturalität der modernen Gesellschaften, d. h. sie vernachlässigt sowohl die kulturelle Diversität des sozialen Geflechts als auch den multiplen oder hybriden Charakter der individuellen Identitäten (vgl. z. B. Welsch 2005). Statt zur Grundlage der Stabilität wird sie zum Quell permanenter Konflikte. Zwar ist der innere Zusammenhalt einer Gesellschaft durch einen ungebremsten Prozess kultureller Fragmentierung gefährdet, doch umgekehrt erstickt eine monolithische kulturelle Struktur jede bürgerliche Spontaneität und Expressivität im Keim. Die nicht bloß konjunkturelle Existenz multikultureller Staaten (im doppelten Verständnis plurinationaler (Schweiz) und polyethnischer (USA) Staaten), die es geschafft haben, eine stabile kollektive Identität auszubilden, zeigt, dass die ethnisch-kulturelle Homogenität der Population keine unverzichtbare Voraussetzung für die Etablierung und Konsolidierung einer politisch-demokratischen Gemeinschaft darstellt. ${ }^{15}$ Diese Überzeugung

15 Dies gilt insbesondere für die Vereinigten Staaten als einer pluralen Nation, welche die Koexistenz unterschiedlicher ethnokultureller Zugehörigkeiten erlaubt. Vgl. hierzu Lacorne 2003. 
wird von Vertretern der politischen Tradition des Republikanismus weitgehend geteilt.

\section{I. Kollektive Identität im republikanischen Modell}

Das republikanische Modell ist mit drei grundlegenden politischen Begriffen verbunden: bürgerliche Partizipation, Primat des Demos und öffentliche Deliberation. Das Wesen des bürgerlichen Republikanismus beruht dementsprechend auf der demokratischen Selbstregierung. Langfristiges Ziel jeder republikanischen Politik ist die Konstruktion eines Vaterlandes, verstanden als Ort der Freiheitsentfaltung (vgl. Viroli 1995). Die Verteidigung der öffentlichen Freiheit, der Freiheit aller, vollzieht sich unter Berufung darauf, dass dies zugleich der persönlichen Freiheit einer jeden in die Gemeinschaft integrierten Person diene. Auf diese Weise hat sich der Republikanismus von Cicero und Titus Livius bis zur Gegenwart, mit Autoren wie Quentin Skinner, Maurizio Viroli oder Philip Pettit, als ein politischer Diskurs artikuliert, der die Selbstverwaltung und Selbstregierung der Bürger verteidigt. Sowohl die alten Republikaner als auch die heutigen Neorepublikaner bekennen sich zur Ablehnung herrschaftlicher Strukturen und zu einer robusten und positiven Freiheitsidee. ${ }^{16}$ Nichtsdestoweniger erlaubt diese entschiedene Stellungnahme ein beachtliches Spektrum von Modulationen. So zeugt die Argumentation Machiavellis (vor allem in seinen Discorsi sopra la prima deca di Tito Livio, I513-1519) nicht nur von einem hoch entwickelten Realismus, sondern zugleich von einem gewissen anthropologischen Pessimismus. Nicht umsonst sind die Republikaner so beharrlich darum besorgt, der Korruption und dem Machtmissbrauch durch politische Institutionen einen Riegel vorzuschieben, da diese die bürgerlichen Tugenden untergraben und die Willkür befördern. ${ }^{17}$

16 Charakteristisches Merkmal einer republikanischen Freiheitskonzeption ist die auf den ersten Blick paradox anmutende enge Verbindung von Freiheit und Gemeinwohl: "We can only hope to enjoy a maximum of our own individual liberty if we do not place that value above the pursuit of the common good." (Skinner 1996, 22I)

17 „Nachdem der militärische Heroismus in der Vergangenheit versunken ist, könnten vielleicht die moralische Entrüstung und Unnachgiebigkeit angesichts des Machtmissbrauchs als republikanische Werte, aber angepasst an die privatisierte Moral unserer Zeit, Geltung erlangen." (Béjar 2003, 36) Dabei ist nicht zu vergessen, dass die bürgerlichen Tugenden die Existenz einer Wertegemeinschaft bzw. eine politische Kultur voraussetzen. In diesem Sinn wurde der Begriff der areté schon im 
Es ist richtig, dass die Republikaner dem stereotypen Bild eines liberalen, egoistischen und berechnenden Individuums den anspruchsvollen Begriff eines Bürgers entgegen setzen, der an das Gemeinwohl glaubt und es dem eigenen, persönlichen Wohl voran stellt. Diese schöne Vision erscheint, vorsichtig gesagt, kontraintuitiv. Und dennoch kann der republikanische Ansatz nicht einfach als naiv oder unrealistisch abgetan werden. Schon die bloße Existenz empirischer Koordinationsprobleme zwischen den politischen Akteuren, auch wenn diese über die beste bürgerliche Gesinnung verfügen sollten, begründet die Notwendigkeit der Vereinbarung von Regeln. Die republikanischen Autoren schenken diesen funktionalen Anforderungen Gehör und überlassen keineswegs alles der guten Veranlagung der Bürger. Davon zeugt „die beständige republikanische Sorge um die Gestaltung von Institutionen, die durchlässig sein sollen für ,die Interessen aller und die es erlauben, alle Äusserungen und Einwände zu berücksichtigen. Nichts davon hätte Sinn in einer idealen Welt unendlich tugendhafter, perfekt informierter und unbegrenzt grosszügiger Bürger." (Ovejero 2003, 22) Gerade weil die republikanischen Autoren sich der Schwäche der Tugend und der ständigen latenten Korruptionsgefahr bewusst sind, weil sie sich ein betont realistisches Bild der menschlichen Natur machen, widmen sie sich so leidenschaftlich dem Entwurf von Kontrollmechanismen und Systemen des Machtausgleichs, ${ }^{18}$ die auch von den liberalen Denkern sehr geschätzt werden. So scheint es möglich, den vom Republikanismus eingeschla-

antiken Griechenland verstanden, als Ausdruck der gesellschaftlichen Anerkennung einer Person für ihr vortreffliches Verhalten im öffentlichen Leben (vgl. MacIntyre 1984).

18 So widmet beispielsweise Philip Pettit (1997, Kap. 6, 7 und 8), einer der originellsten zeitgenössischen Vertreter des Republikanismus, einen beträchtlichen Teil seiner bekanntesten Monographie (in der er einen republikanischen Freiheitsbegriff im Sinne der non-domination, des Schutzes vor willkürlichen Eingriffen, vertritt) der Forderung nach einer ganzen Reihe von Mechanismen, Klauseln und Hürden zur Prävention und Verhinderung des Machtmissbrauchs, der Tyrranei durch die Mehrheit und der Korruption der Regierenden. Im übrigen gibt es guten Grund davon auszugehen, dass einige der heute als liberal abgestempelten Mechanismen in Wirklichkeit genuin republikanischer Herkunft sind, eine Hypothese, die bestätigt wird durch Skinners (1996, 215 f.) Interpretation des Republikanismus als „a strongly contrasting way of thinking about the relations between liberty and the common good, one that not only predates modern liberalism but has largely been obliterated by its triumph". Um es mit Savidan (2003, 136) zu sagen: die republikanische Tradition „hat lange Zeit unter der Tatsache gelitten, dass sie von der liberalen Neufassung überlagert wurde“. 
genen Pfad weiter zu verfolgen, ohne den vom Liberalismus eröffneten Weg ganz zu verlassen. ${ }^{19}$

Der Begriff des Vaterlandes, so wie er vom Republikanismus geprägt ist, hat unbestreitbar positive Konnotationen, auch wenn sich im alltagssprachlichen Gebrauch ein zunehmend negatives Verständnis geformt hat, so dass wahrscheinlich viele Leser eine Politik der Exklusion damit verbinden. ${ }^{20}$ Die republikanische Tradition hat darüber hinaus einen starken Begriff von Bürgerschaft entwickelt und betont die enorme Bedeutung der Gesetze und öffentlichen Institutionen, welche die Ausübung bürgerlicher Freiheiten erst ermöglichen. Durch Ausweitung des Bürgerstatus sucht sie, eine kollektive Identität zu konfigurieren, die auf der verantwortlichen Partizipation der Individuen in öffentlichen Angelegenheiten basiert, da „die Nicht-Ausübung der positiven Freiheiten zu einer Fragilisierung der negativen Freiheiten führ[e]" (Sadivan 2003, 157). Der Bürgerstatus sichert die soziale Kohäsion und die Identifikation der Personen mit dem Staat. ${ }^{21}$ Die Beteiligung der Bürger an der politischen

19 In der Linie einer Deutung, die davon ausgeht, dass „,modern liberalism is the heir of the republican tradition, which it further develops along individualistic lines, and not the other way around“ (Ferrara 2008, 108), finden sich Ausdrücke wie „republikanischer Liberalismus“ oder „liberaler Republikanismus“, um Spielarten des Republikanismus zu beschreiben, die sich nicht als Alternative zum Liberalismus verstehen, sondern als integrative Modelle oder Formen eines korrigierten Liberalismus. John Rawls (200I, I42-I45) beispielsweise ist der Überzeugung, dass Liberalismus und Republikanismus durchaus kompatibel sind, sofern man unter Letzterem die Forderung nach aktiver Beteiligung der Bürger am öffentlichen Leben versteht (so deutet er selbst den klassischen Republikanismus). Zugleich macht er deutlich, dass beide Konzeptionen in offenen Widerspruch geraten, wenn man beim Republikanismus an die politische Partizipation als Lebensform denkt und dies zur umfassenden Doktrin macht. In diesem Fall ist es Rawls zufolge angebrachter, von bürgerlichem Humanismus zu sprechen. Sowohl der politische Liberalismus als auch der klassische Republikanismus verstehen die bürgerliche Partizipation als Mechanismus zur Verteidigung von Freiheit und Gerechtigkeit, während der bürgerliche Humanismus more aristotelico - daraus den priviligierten Ort eines erfüllten menschlichen Lebens macht.

20 Die negativen Konnotationen sind wohl unter anderem darauf zurückzuführen, dass der republikanische Patriotismus im alltäglichen Sprachgebrauch oftmals mit dem Nationalismus gleichgesetzt wird. In Wirklichkeit besteht zwischen beiden Ideologien aber kein streng begrifflicher Nexus, sondern lediglich eine zufällige historische Verbindung (vgl. Habermas I998, 619-643). Tatsächlich haben die nationalistischen Bewegungen, vor allem seit dem 19. Jahrhundert, die normative Bedeutung des Republikanismus verwässert und die Liebe zu den geteilten Freiheiten in eine Verehrung der ethnokulturellen Einheit und Reinheit verwandelt (vgl. Viroli 1995).

21 Inwieweit die Zuerkennung der Staatsbürgerschaft als Mechanismus der sozialen 
Macht verstärkt das Gefühl der Zugehörigkeit zum Staat: „le plus puissant moyen, et peut-être le seul qui nous reste, d'intéresser les hommes au sort de leur patrie, c'est“, wie Tocqueville (I98I, Bd. I, 33I) betont, „de les faire participer à son gouvernement". Das Vaterland des Republikaners ist nicht nur ein durch gemeinschaftliche Erinnerung und gemeinsame Sitten geformter Ort, sondern ein Raum der Partizipation Aller an den öffentlichen Angelegenheiten. So überrascht es nicht, dass der Republikanismus die Politik als natürliches Mittel der demokratischen Selbstverwaltung auszeichnet. Das republikanische Ideal ist untrennbar verbunden mit der Ausübung der Demokratie, der Partizipation an öffentlichen Deliberationen, der Forderung nach Öffentlichkeit und Transparenz in den Verfahren und der kollektiven Kontrolle der Autoritäten. ${ }^{22}$

Der Patriotismus, d. h. diejenige kollektive Identität, die sich aus einer republikanischen Praxis ableitet, ist durch und durch bürgerlicher Natur und ruht auf keinerlei ethnisch-kulturellen Komponenten. ${ }^{23}$ Auch wenn einige Texte des modernen Republikanismus (besonders bei Machiavelli und Rousseau) nicht gerade ein pluralistisches und inklusives Panorama entwerfen, so lässt sich doch auf der Basis republikanischer Ansätze sehr gut mit dem Phänomen der multiplen kulturellen Zugehörigkeit umgehen. Das Profil des guten Staatsbürgers republikanischer Prägung ist das eines offenen und pluralen Individuums, denn es stützt sich auf keine nicht-wählbaren Elemente, sondern auf das Prinzip der multiplen und freiwilligen Mitgliedschaft. Letzten Endes wird von dem Bürger nichts erwartet als Loyalität zu den demokratischen Prinzipien, auf denen der Staat errichtet ist, jedoch „keinerlei Identifikation mit irgendwelchen ethnokulturellen Inhalten" (Colom 1998, 83). ${ }^{24}$

Kohäsion und Integration in Einwanderungsländern wie Deutschland funktioniert, ist eine offene Frage. Zwar garantiert die Einbürgerung dauerhaft angesiedelter Immigranten noch nicht deren Integration, aber sie stellt eine unverzichtbare Voraussetzung für diese dar und unterbindet zudem typische Formen der Marginalisierung.

22 Trotz der traditionell engen Verbindung von Republikanismus und Demokratie hat es auch republikanische Strömungen gegeben, die sich nicht mit der Demokratie identifizierten. Allerdings hat dies wohl vor allem mit der historischen Entwicklung des Demokratiebegriffs zu tun. Vgl. Rivero 2005.

23 Das Streben nach kultureller Homogenität ist vielmehr ein charakteristisches Merkmal des ethnisch-kulturellen Nationalismus, nicht aber des Republikanismus. Eine aufschlussreiche Studie zu den Unterschieden zwischen Nationalismus und Republikanismus liefert Viroli 1995 .

24 Auch in einer Republik ist die systematische Exklusion ganzer Bevölkerungsschichten keineswegs ausgeschlossen. Ein paradigmatisches Beispiel hierfür ist die grie- 
Natürlich ist es für den Umgang mit den Problemen einer multikulturellen Gesellschaft angezeigt, neue Überlegungen in das politische Modell des klassischen Republikanismus einzubringen. In einer kulturell pluralen Gesellschaft werden die Bürger große Schwierigkeiten haben, sich mit einem Staat zu identifizieren, dessen Institutionen zwar die individuellen Rechte und Freiheiten schützen, sich jedoch in keiner Weise für die Kulturen einsetzen, denen sich die Bürger zugehörig fühlen. Nur wenn der Staat ausreichend politische Sensibilität für das kulturelle Mosaik aufbringt, können auch die nicht der Mehrheit angehörigen Bürger Loyalität gegenüber dem Staat zeigen, ohne dabei in Konflikt mit ihrer besonderen Identität zu geraten. Anderenfalls muss man sich die Frage stellen, wie man Loyalität verlangen kann von jemandem, dem es z. B. nicht erlaubt ist oder nicht ermöglicht wird, sich in seiner eigenen Sprache auszudrücken, gesellschaftlich zu engagieren und politisch zu partizipieren. ${ }^{25}$ Aus diesem Grunde kann ein im engen Sinne plurinationaler oder pluriethnischer Staat nur dann Beitritt und Zustimmung von seinen Bürgern fordern, wenn irgendeine institutionalisierte Form des Umgangs mit der Differenz gefunden ist. Diese Aufgabe ist einfacher, wenn das Leben in der Gemeinschaft auf einem breiten konstitutionellen Konsens aufruht, der die Existenz unterschiedlicher ethnischer und kultureller Gemeinschaften anerkennt und nicht nur ein System wechselseitiger Solidarität etabliert, sondern so weit als möglich das Fortbestehen der kulturellen Traditionen gewährleistet.

Doch mit der staatlichen Unterstützung des Fortbestandes unterschiedlicher Kulturen und gemeinschaftlicher Praktiken ist das Problem noch nicht gelöst. Es gilt, eine stabile Grundlage der Solidarität zu kreieren, ohne die ein friedliches Zusammenleben multipler kollektiver Iden-

chische Polis, in der Metöken, Sklaven und Frauen von den öffentlichen Angelegenheiten ausgeschlossen waren. Nichtsdestoweniger zielen die republikanischen Prinzipien im Kern auf eine Inklusion der gesamten Bevölkerung eines Territoriums. Die Leitprinzipien der französischen Republik sind hierfür beispielhaft. Die Qualität einer Republik bemisst sich am Grad der Aufmerksamkeit, die den Ausgeschlossenen gewidmet wird, an dem Grad, in dem die soziale Marginalisierung bestimmter Gruppen verhindert wird.

25 Diese Überlegung gilt in erster Linie für die plurinationalen Staaten, in denen die ethnischen oder kulturellen Gruppen mehrheitlich bestimmte Territorien bevölkern. Für den Fall der multikulturellen Staaten mit verstreuten Minderheiten, wie sie vor allem durch Einwanderung entstehen, bleibt das Erlernen der offiziellen Landessprache ein ganz wesentlicher Faktor für die Integration und darüber hinaus Voraussetzung für eine mögliche politische Partizipation. 
titäten kaum möglich erscheint. Die durch den Pluralismus der Werte und kollektiven Identitäten gekennzeichneten Gesellschaften hätten keinen Zusammenhalt ohne gemeinsame Prinzipien, welche die Interaktion der unterschiedlichen Gruppen regeln, und ohne eine gemeinsame politische Identität jenseits der bestehenden Unterschiede. Der kulturelle und axiologische Pluralismus kann niemals so radikal sein, dass er jegliche Mechanismen der Koordination und jegliche gemeinschaftliche Grundlage der wechselseitigen Anerkennung ausschließt.

Soziale Integration wird in den pluralen modernen Staaten auf zwei Weisen realisiert: als sittlich-kulturelle und/oder als politisch-kulturelle Integration (vgl. Thiebaut 1998, I4I-I6I). Die erste Form der Integration vollzieht sich im lebensweltlichen Umfeld und bezieht sich auf Traditionen, Bräuche, Praktiken und Lebensformen. Die zweite ist abstrakter und bezieht sich im Kern auf diejenigen Werte und Prinzipien, die das friedliche Zusammenleben ermöglichen. Der demokratische Rechtsstaat ist so konzipiert, dass die Ebene der allgemeinen politischen Kultur, zu der sich alle Bürger bekennen müssen, abgekoppelt sein sollte von der Ebene spezifischer ethnisch-kultureller Integration. Ethnisch-kulturelle Identität und bürgerliches Bewusstsein dürfen nicht über einen Kamm geschoren werden.

Die bürgerlich-demokratische Kultur desaktiviert teilweise das ausschließende und partikularistische Potential bestimmter Lebensformen, gesteht ihnen aber einen adäquaten Rahmen für ihre Entfaltung zu. Folglich sollte ein republikanischer Staat von seinen Bürgern und von denjenigen, die sich willentlich entschieden haben, in ihm zu leben, d. h. von den Immigranten und Asylanten, lediglich eine politische Akkulturation fordern. Er sollte keine weiter reichenden Forderungen aufstellen, da die Erhaltung der kollektiven Identität einer demokratischen Gemeinschaft nicht erfordert, dass alle Individuen bestimmte kulturelle Traditionen teilen. Unter Einhaltung dieser Richtlinien sollte die Errichtung eines laizistischen öffentlichen Raumes möglich sein, eines bürgerlichen Raumes gemeinsam geteilter Werte und Prinzipien.

Um die praktischen Implikationen unserer Ausführungen zu einem republikanischen Identitätsgefühl anschaulich zu machen, sei auf zwei historische Ereignisse verwiesen: den Watergate-Skandal und die terroristischen Anschläge des II. September. Das Zusammengehörigkeitsgefühl, das sich in der nordamerikanischen Gesellschaft in Reaktion auf die Terroranschläge schlagartig ausgebreitet hat, stellt eine spontane Reaktion auf die direkte, gegen die Gesellschaft gerichtete Aggression dar. 
Das so provozierte Identitätsgefühl resultiert aber nicht unbedingt aus einer republikanischen Identifikation. Demgegenüber gibt es subtilere Angriffe auf die Gesellschaft, die sich darauf richten, das soziale Netz zu zerstören, wie beispielsweise im Fall Watergate die Verletzung grundlegender Normen, auf denen das Vertrauen in die Regierung beruht. Um diese aufzudecken bedarf es einer ausgeprägten staatsbürgerlichen Sensibilität (vgl. Taylor 1995, 195 ff.). Das mit dem Begriff des Patriotismus intern verbundene Ideal der Selbstregierung hat eine vitale Funktion hinsichtlich der Verteidigung der Freiheiten in einer Gesellschaft. Es entsteht aus der Identifikation der freien Bürger mit den Interessen der Gemeinschaft, in der sie leben, in letzter Konsequenz mit dem gesellschaftlichen Gemeinwohl. So ist es nicht verwunderlich, dass sich das patriotische Gefühl ausdrückt als Zufriedenheit oder Stolz über die Mitwirkung an einem gemeinschaftlichen Unternehmen und als Solidarität angesichts gemeinschaftlich erfahrenen Unglücks. Doch die wohl genuinste und reifste Form der Manifestation eines solchen Gefühls ist die der Indignation, der Empörung über den Machtmissbrauch durch Regierung und Behörden oder über die Korruption der Institutionen. Der Patriotismus republikanischen Zuschnitts ist grundlegend mit der staatsbürgerlichen Kultur verbunden und mit einem vitalen Interesse an der politischen Bildung und Erziehung (nicht aber Indoktrination) in der demokratischen und pluralistischen Gesellschaft.

\subsection{Institutionelle Implikationen: ein offenes verfassungsstaatliches Modell}

Aus den republikanischen Prämissen der Inklusion und der bürgerlichen Beteiligung lassen sich einige für den Zuschnitt der politischen Gemeinschaft nicht unbedeutende Folgerungen ableiten. Zunächst einmal müssen, um Diskriminierung zu vermeiden, die Erfordernisse für das Erlangen der Staatsbürgerschaft, verstanden als Status der abstrakten Gleichstellung aller Individuen, durch klare und durchsichtige Verfahren geregelt sein. Die einzige unabdingbare Voraussetzung, um eine Person als volles Rechtssubjekt in einem republikanischen Staat anzuerkennen, sollte deren ausdrückliche Zustimmung zur Verfassung und zu der in Kraft befindlichen Rechtsordnung sein, niemals aber die vollständige Anpassung an die so genannte Nationalkultur. So wäre es unangebracht, das Bekenntnis zur mehrheitlichen Religion zur Kondition für die Zuerkennung aller Rechte eines Mitglieds der Gemeinschaft zu 
machen. Auch erscheint es nicht unbedingt notwendig, ausdrücklich das Erlernen der offiziellen Landessprache zu fordern, wenngleich dies dem Anwärter auf Einbürgerung natürlich in jedem Fall zum Vorteil gereicht. Nur allzu oft hört man das Vorurteil, dass die Immigranten nicht bereit seien, sich gegenüber dem Einwanderungsland zu öffnen. Es fällt schwer, den angemessenen Respekt gegenüber der Person und ihrer Identität auch gleich mit ihrer Integration als vollwertiges Mitglied der Gesellschaft zu verbinden. Um sich als Staatsbürger zu verstehen und als solcher zu leben reicht es aus, die Richtlinien zu kennen und einzuhalten, welche die Teilnahme am politischen Leben der Gemeinschaft ermöglichen. Sofern sie sich als attraktiv und gut darstellen, werden die Sitten und kulturellen Praktiken des Aufnahmelandes in der Regel ohne Ausübung von Zwang übernommen. ${ }^{26}$ Umgekehrt hat sich die Präsenz von Minderheiten in der Regel eher als bereichernd und stimulierend für die Mehrheitskultur erwiesen. Als Bedrohung wird sie lediglich von denjenigen empfunden, die den Wert der eigenen Kultur nicht hinreichend schätzen und sich in der Begegnung mit fremden Kulturen angegriffen oder beeinträchtigt fühlen.

Sicherlich löst der Zugang zur Staatsbürgerschaft nicht alle Probleme der Minderheiten. Im Falle verstreuter ethnischer Minderheiten oder von Immigrantengruppen ohne klare territoriale Zuordnung gilt es zu vermeiden, dass ihre Verschiedenheit als Motiv für soziale Diskriminierung herangezogen wird. Mögliche Gegenmaßnahmen sind wesentlich anti-diskriminatorischer Natur, also darauf gerichtet, eine effektive Gleichbehandlung vor dem Gesetz zu erzielen. Im Gegensatz dazu stößt der Rekurs auf positive Diskriminierung und Affirmative Actions in der Regel auf Widerstand und erfordert eine beachtliche pädagogische Anstrengung. Der kompensatorische Zweck dieser Maßnahmen muss ausreichend klar sein, so dass sie nicht als Privilegien missverstanden werden (vgl. Velasco 2007).

26 Diese Auffassung wird von Vertretern anderer politischer Ansätze geteilt, so z. B. von Joseph Raz (1995, I82): „Liberal multiculturalism is not opposed in principle to the assimilation of one cultural group by others. In some countries some of the constituent cultures may lose their vitality and be gradually absorbed by others. So long as the process is not coerced, does not arise out of lack of respect for people and their communities, and is gradual, there is nothing wrong in it. The dying of cultures is as much part of normal life as the birth of new ones." Anders liegt der Fall natürlich, wie Sartori (2000) deutlich gemacht hat, wenn man es mit Gruppen von Immigranten zu tun hat, die überzeugte Gegner einer demokratischen Kultur sind. 
Ähnliche Vorschläge zum Umgang mit dem Problem des Kulturpluralismus sind von verschiedenen Autoren vorgelegt worden. Neben Habermas (1996, 237-276) hat der britische Soziologe John Rex einschlägige Überlegungen präsentiert. Er vertritt die Auffassung, dass eine unter demokratischem Gesichtspunkt akzeptable multikulturelle Alternative die Existenz zweier klar unterschiedener kultureller Bereiche impliziert. Die Position ist daher als Two-Domains-Thesis (Rex 2004, 40-46) bekannt geworden: „On the one hand there was, or ought to be, a shared public political culture which laid down the basic political 'rules of the game' [...]. On the other, there should be an acceptance of a variety of communal cultures involving the right of members of separate communities to speak their own mother tongue, to follow their own religions and to have their own customs and family practices" (Rex I996, I34). Zur Aushandlung der Grenzen zwischen den beiden Bereichen ist es wichtig „[to] leave open a space for dialogue“ (Rex 2004, 42). In eine ähnliche Richtung zielt der liberale Denker und Rechtstheoretiker Joseph Raz, wenn er betont, dass ein multikultureller Staat aus verschiedenen Gemeinschaften bestehe, aber keiner von ihnen angehöre, so dass Elemente der Kohäsion gefunden werden müssen, „a common culture in which the different coexisting cultures are embedded" (Raz 1995, I87). Dies erfordert, dass die Mitglieder der verschiedenen kulturellen Gemeinschaften eine gemeinsame politische Sprache und Verhaltenskonventionen übernehmen, die es ihnen erlauben „to compete effectively for resources and to be able to protect their group as well as individual interests in a shared political arena" (Raz 1995, I88). ${ }^{27}$ Eine gemeinsame politische Kultur sollte keine Hürde, sondern Bedingung der Möglichkeit sein, um die angewachsenen kulturellen Ansprüche und Forderungen geltend zu machen, die nicht mehr nur die freie Ausübung der differenten kulturellen Praktiken im privaten Raum, also Toleranz, einfordern, sondern Ausdruck, Präsenz und Anerkennung im öffentlichen Raum anstreben (vgl. Maclure/Taylor 20IO, 44).

Um das republikanische Modell in einem multinationalen und multiethnischen Kontext effektiv umzusetzen, müssen Einigungen auf Verfassungsebene getroffen werden. Und es ist ein flexibleres Verständnis der Verfassung erforderlich als in der rigiden kontinental-europäischen

27 Mit einer solchen Unterscheidung von Sphären - die nicht nur von Habermas, Rex und Raz, sondern auch von Walzer vertreten wird - sollen Extremismen vermieden werden, die das gemeinschaftliche Leben unmöglich machen. Zum Verhältnis dieser Sphären vgl. auch Martiniello (I998, 89). 
Tradition. Sie ist dynamisch als ein immer unvollendetes Projekt ohne essentialistische Züge aufzufassen (vgl. Habermas 1992, 464). Sie sollte als „offenes Werk “, als notwendig fehlbar und somit revidierbar, verstanden werden, nicht als totes „historisches Dokument“, sondern als Projekt einer gerechten Gesellschaft, das den "Erwartungshorizont" einer politischen Gemeinschaft abbildet, der von allen Mitgliedern durch ihre Interpretationen schrittweise an die sozialen Veränderungen angepasst wird. ${ }^{28}$ Es sollte nicht darum gehen, die Wirklichkeit an die Verfassungsnorm anzupassen und das politische System künstlich abzuriegeln, sondern das System zu öffnen und unter gewissen Maßgaben an die sozialen Verhältnisse anzupassen. Auf der Basis einer so gestalteten Verfassung erscheint eine Kompatibilität der vielfältigen individuellen und kollektiven Identitäten mit einer gemeinsamen politischen und konstitutionellen Loyalität möglich. Die Vervielfachung der Identitäten, in der Gesellschaft und in den einzelnen Subjekten, stellt keineswegs ein Hindernis für die soziale Integration dar. Im Gegenteil, mit der Vermehrung der Identitäten teilen sich auch die Leidenschaften, Vorlieben und Interessen auf (identities multiply - passions divide) (vgl. Walzer 1994, 82), wodurch sich das politische Aktionsfeld, der Raum für Verhandlung, Dialog und mögliche Übereinkunft, erweitert.

Nichtsdestoweniger ist eine gewisse Reserviertheit gegenüber dem skizzierten Vorschlag verständlich, insbesondere hinsichtlich der Fragen, ob das hier nur angedeutete republikanische Modell die politische Imagination der Bürger zu erfassen vermag, ob die freiwillige Zustimmung zu den genannten Grundsätzen eine ausreichende Grundlage für die Generierung kollektiver Identitäten bildet und nicht nur eine aseptische Beziehung zu einer administrativen Entität konstituiert, sondern soziale Integration gewährleistet, die das Leben in der Gemeinschaft für alle attraktiv erscheinen lässt.

Die Verteidigung eines bürgerlichen Republikanismus ist als reflektierte Reaktion auf die Bestrebungen zu verstehen, um jeden Preis eine Homogenisierung der Gesellschaft zu erreichen und die soziale und kul-

28 Weit davon entfernt, bloße Empfänger der Normen zu sein, bilden in diesem Modell die Bürger, und nicht nur die qualifizierten juristischen Operatoren die, wie sie Peter Häberle (1998, I58-I8I) treffend benennt, „offene Gesellschaft der Verfassungsinterpreten". Die Interpretation der Verfassung ist dabei in Abhängigkeit von der Verwurzelung und Vitalität der bürgerlichen Kultur und vom Grad der Beteiligung und des Engagements der Bürger im öffentlichen Raum als „offener und pluraler“ Prozess konzipiert. 
turelle Pluralität soweit wie möglich zu reduzieren. Sie stellt eine plausible Antwort unter demokratischen Bedingungen dar. Zentraler Bestandteil des Entwurfes ist die Identifikation mit einem Staat, der sich auf die unparteiliche Anwendung des demokratischen Rechts verpflichtet und zugleich das Recht auf abweichende nationale Identifikation und abweichende Auffassungen bezüglich der zukünftigen Gestaltung der politischen Gemeinschaft garantiert. Ein solcher Republikanismus erfordert das Eintreten für demokratische Grundsätze der bürgerlichen Integration und des interethnischen Zusammenlebens basierend auf der Idee der Zwangsvermeidung (no imposition), auf dem Respekt vor der Alterität und auf der Einstufung der kulturellen Diversität als grundlegendes Recht aller Individuen. Verglichen mit dem Nationalismus präsentiert sich ein so verstandener Republikanismus als offener, inklusiver und sensibler für die multiplen Identifikationen; verglichen mit dem Liberalismus zeigt er ein größeres Interesse an der Verfestigung und Absicherung der sozialen Integration innerhalb der politischen Gemeinschaft.

Ein republikanisches Politikverständnis kann legitimer Weise Anspruch darauf erheben, als Prinzip der Kohäsion in einer pluralistischen Gesellschaft zu gelten, d. h. als kleinster gemeinsamer Nenner, auf den sich ansonsten disparate ideologische und kulturelle Positionen einigen könnten. Das bedeutet aber nicht, dass für bestimmte im Kern multinationale Gesellschaften - wie in Kanada, Belgien oder Spanien nicht auch die Ausbildung eines Patriotismus der Pluralität zweckmäßig wäre, der nicht nur die grundlegende Loyalität zur Verfassung voraussetzt, sondern sich in einem klaren Bewusstsein der Diversität niederschlägt und die bestehenden identitären Affekte überlagert. Der wichtigste Beitrag, den eine republikanische Pädagogik der Pluralität leisten könnte, bestünde darin, bei den Staatsbürgern in kohärenter Weise ein Zugehörigkeitsgefühle zu einer Gemeinschaft zu erzeugen, die sich klar und öffentlich zu einer multinationalen und pluriethnischen Kultur bekennt.

\section{Literatur}

Anderson, Benedict 1991: Imagined communities, London.

Beiner, Ronald 2003: Liberalism, Nationalism and Citizenship, Vancouver.

Béjar, Helena 2003: "La sociología de la libertad de J. G. A. Pocock", in: Claves de razón práctica 13I, 33-39. 
Benhabib, Seyla 1999: Kulturelle Vielfalt und demokratische Gleichheit, Frankfurt a. M.

Colom, Francisco 1998: Razones de identidad, Barcelona.

Cossío, José Ramón 2000: "Constitucionalismo y multiculturalismo", in: Isonomía I2, 75-93.

Dion, Stéphane 1996: "Bélgica y Canadá: comparación de sus oportunidades de supervivencia", in: Debats 56, 17-37.

Dworkin, Ronald I990: "Foundations of Liberal Equality", in: The Tanner Lectures on Human Values XI, Salt Lake City, I-II9.

Ferrara, Alessandro 2008: The Force of the Example, New York.

Fetscher, Iring 1990: Toleranz, Stuttgart.

Forst, Rainer 2003: Toleranz im Konflikt, Frankfurt a. M.

Garzón Valdés, Ernesto 2000: Instituciones suicidas, México.

Gellner, Ernst 1987: Culture, Identity, and Politics, Cambridge.

Giner, Salvador 2000: "Cultura republicana y política del prevenir", in: S. Giner (Hg.), La cultura de la democracia, Barcelona, I37-174.

Gray, John 2000: Two Faces of Liberalism, New York.

Häberle, Peter 1998: Verfassung als öffentlicher Prozess, Berlin.

Habermas, Jürgen 1992: Faktizität und Geltung, Frankfurt a. M.

- 1996: Die Einbeziehung des Anderen, Frankfurt a. M.

- 2005: Zwischen Naturalismus und Religion, Frankfurt a. M.

Höffe, Otfried 1999: Demokratie im Zeitalter der Globalisierung, München.

Ignatieff, Michael 20or: Human Rights as Politics and Idolatry, Princeton.

Lacorne, Denis 2003: La crise de l'identité américaine, Paris.

Kymlicka, Will 1995: Multicultural Citizenship, Oxford.

- 200I: Politics in the Vernacular, Oxford.

- 2007: Multicultural Odysseys, Oxford.

MacIntyre, Alasdair 1984: After Virtue, Notre Dame.

Maclure, Jocelyn/Taylor, Charles 20ıо: Laïcité et liberté de conscience, Montréal.

Martinello, Marco 1998: Salir de los guetos culturales, Barcelona.

Miller, David 1995: On Nationality, Oxford.

Ovejero, Félix 2003: "Tres miradas republicanas sobre la democracia”, in: Claves de razón práctica $132,20-30$.

Parekh, Bhikhu 2000: Rethinking multiculturalism, London.

- (2008): A new politics of identity, Houndmills; New York.

Pettit, Philip 1997: Republicanism, Oxford.

Pocock, J. G. A. 2003: The Machiavellian Moment, Princeton.

Rawls, John 1993: Political Liberalism, New York.

- 200I: Justice as fairness: a restatement, Cambridge.

Raz, Joseph 1995: "Multiculturalism: A Liberal Perspective", in: Ethics in the Public Domain, Oxford, 170-192.

Requejo, Ferran 2000: "El liberalismo político en Estados plurinacionales", in: J.-F. Prud'homme (Hg.): Demócratas, liberales y republicanos, Mexiko, 43-72. 
Rex, John 1996: Ethnic Minorities in the modern Nation State, London.

- 2002: "Multiculturalism and Political Integration in the Modern NationState", in: J. Rex y G. Singh (Hg.) Governance in Multicultural Societies, Aldershot, 36-48.

Rivero, Ángel 2005: "Republicanismo y neo-republicanismo", in: Isegoría 33, $5-17$.

Sartori, Giovanni 2000: Pluralismo, multiculturalismo e estranei, Mailand.

Savidan, Patrick 2003: "La crítica republicana del liberalismo", in: J. Conill, D. A. Crocker (Hgs.): Republicanismo y educación cívica, Granada, I35-I58.

Skinner, Quentin 1992: "On Justice, the Common Good, and the Priority of Liberty”, in: Ch. Mouffe (Hg.), Dimensions of Radical Democracy, London, 2II-224.

Tamir, Yael 1993: Liberal nationalism, Princeton NJ.

Taylor, Charles I992: Multikulturalismus und die Politik der Anerkennung, Frankfurt a. M.

- 1995: Philosophical Arguments, Cambridge MA.

Tocqueville, Alexis de i98r: De la Démocratie en Amérique, Paris.

Thiebaut, Carlos i998: Vindicación del ciudadano, Barcelona.

- I999: De la tolerancia, Madrid.

Tully, James 1995: Strange multiplicity, Cambridge.

Velasco, Juan Carlos 2005: "La noción republicana de ciudadanía y la diversidad cultural", in: Isegoría 33, I9I-206.

- 2007: "Discriminación positiva, diversidad cultural y justicia", in: Daimon $4 \mathrm{I}, \mathrm{I} 43-\mathrm{I} 58$.

Villoro, Luisi998: Estado plural, pluralidad de culturas, Mexiko.

Viroli, Maurizio 1995: For Love of Country, Oxford.

Wagner, Astrid 2013: „Globalización y diversidad cultural”, in: D. Brauer (Hg.), Pensar la globalización, Buenos Aires (im Erscheinen).

Walzer, Michael 1984: „Liberalism and the Art of Separation“, in: Political Theory I2, 315-330.

- 1994: Thick and Thin, Notre Dame.

Welsch, Wolfgang 2005: „Transkulturelle Gesellschaften“, in: P. U. Merz-Benz, G. Wagner (Hgs.), Kultur in Zeiten der Globalisierung, Frankfurt a.M., $39-67$. 